\title{
Quark-antiquark asymmetry of helicity distributions in the nucleon sea
}

\author{
Mengyun Liu ${ }^{1}$ and Bo-Qiang Ma ${ }^{1,2,3, *}$ \\ ${ }^{1}$ School of Physics and State Key Laboratory of Nuclear Physics and Technology, Peking University, \\ Beijing 100871, China \\ ${ }^{2}$ Collaborative Innovation Center of Quantum Matter, Beijing, China \\ ${ }^{3}$ Center for High Energy Physics, Peking University, Beijing 100871, China
}

(Received 12 July 2018; published 30 August 2018)

\begin{abstract}
We study the helicity distributions of light flavor quark-antiquark $(q \bar{q})$ pairs in the nucleon sea. The valence quarks are handled by adopting the light-cone SU(6) quark-spectator-diquark model and the sea $q \bar{q}$ pairs are treated from statistical consideration by introducing the helicity suppression factors $l_{q}(x)$ and $\bar{l}_{q}(x)$ to parametrize the helicity distributions of q-flavor sea quark and antiquark respectively, while $\Delta l_{q}(x)=l_{q}(x)-\bar{l}_{q}(x)$ represents a combined effect of helicity contribution due to sea $q \bar{q}$ pairs. From fitting the nucleon polarization asymmetries $A_{1}^{N}$ in inclusive deep inelastic scattering processes and the single-spin asymmetries $A_{L}^{W^{ \pm}}$in Drell-Yan type processes, we find a significant asymmetry between the quark and antiquark helicity distributions of the nucleon sea. Therefore the quark-antiquark asymmetry of helicity distributions of nucleon sea $q \bar{q}$ pairs, i.e., $\Delta q_{s}(x) \neq \Delta \bar{q}_{s}(x)$, plays an important role for a comprehensive understanding of the nucleon spin content.
\end{abstract}

DOI: 10.1103/PhysRevD.98.036024

\section{INTRODUCTION}

The spin structure of hadrons has received considerable attention since the so-called "proton spin crisis" [1,2], which implies that only a small fraction (about 30\% in recent studies [3-5]) of the proton spin comes from quark spins. Such a small quark spin contribution seems to contradict with the naive quark model [6,7] where all of the proton spin is provided by valence quark spins. However, it was pointed out in Refs. [8-11] that the quark helicity observed in polarized deep inelastic scattering (DIS) is actually the quark spin defined in light-form dynamics [12] and it is different from that in the quark model, which is defined in instant-form dynamics. Therefore the small helicity sum observed by the experiments is not in conflict with the quark model due to the reduction of the light-cone spin relative to the instant-form spin by the Melosh-Wigner rotation [13-15], which is a relativistic effect of quark transversal motions. Calculations of the helicity distributions by valence quarks in the lightcone quark-spectator-diquark model $[10,16]$ can reasonably reproduce the bulk features of the experimental data of

\footnotetext{
"mabq@pku.edu.cn
}

Published by the American Physical Society under the terms of the Creative Commons Attribution 4.0 International license. Further distribution of this work must maintain attribution to the author(s) and the published article's title, journal citation, and DOI. Funded by SCOAP. spin asymmetries on proton [1,17-19], neutron [20], and deuteron [17-19] targets in inclusive DIS processes.

Nevertheless, the sea quark spin contribution to the proton is believed to be nontrivial, as reflected in the recent observations [21,22] of single spin asymmetries of $W^{ \pm}$ production in polarized proton-proton collisions [23,24]. It is shown [21] that a reasonable description of $W^{ \pm}$single spin asymmetry data [23] can be achieved by adopting sizable helicity distributions of the light-flavor up (u) and down (d) antiquarks. More explicitly, the antiquark helicity is positive for the $\bar{u}$ quark $(\Delta \bar{u}>0)$ and negative for the $\bar{d}$ quark $(\Delta \bar{d}<0)$. By adopting the quark-antiquark symmetry of the momentum and helicity distributions between quarkantiquark $(q \bar{q})$ pairs of the nucleon sea, it is found that the Melosh-Wigner rotation effect [8-11] should be much stronger (with larger quark transversal motions) for the valence quarks to reconcile with both data from inclusive DIS processes [25] and $W^{ \pm}$productions [23].

From theoretical considerations, the quark and antiquark of the nucleon sea do not to be symmetric due to the nonperturbative nature of strong interaction. For example, the strange-antistrange asymmetry of the nucleon sea [26-29] has been discussed to study the nucleon strange magnetic moment [30], the NuTeV anomaly [31-36], strange distribution functions of the nucleon [37-39], nucleon form factors $[40,41]$, the direct production of D-meson [42-45], and the $\Lambda / \bar{\Lambda}$ polarization [46-48]. However, the situation becomes subtle for the quark-antiquark asymmetry of the light-flavor sea $q \bar{q}$ pairs because of the existence of the 
valence $\mathrm{u}$ and $\mathrm{d}$ quarks in the nucleon, as one may always define $u_{v}(x)=u(x)-\bar{u}(x)$ and $d_{v}(x)=d(x)-\bar{d}(x)$ as the valence part of the total $u(x)=u_{v}(x)+u_{s}(x)$ and $d(x)=$ $d_{v}(x)+d_{s}(x)$ quark contributions with the assumption of symmetric quark and antiquark momentum distributions: $u_{s}(x)=\bar{u}(x)$ and $d_{s}(x)=\bar{d}(x)$. The same situation also occurs for the helicity distributions of the light-flavor sea quarks. Inspired by the strange-antistrange asymmetry of the nucleon sea of previous studies, we now introduce a quarkantiquark asymmetry of helicity distributions of the nucleon sea while keeping a quark-antiquark symmetry of the momentum distributions for simplicity. We show that the quark-antiquark asymmetry can provide us an interesting scenario of the nucleon sea for a simultaneous description of both experimental data from DIS processes $[1,18-20,49,50]$ and $W^{ \pm}$productions [23].

In this work, we investigate the quark-antiquark asymmetry of helicity distributions of the nucleon sea with the valence quarks handled by the light-cone SU(6) quarkspectator-diquark model $[10,11,51]$ and the sea quarks treated by introducing new parameters from statistical consideration. Numerical results are presented by fitting both the nucleon polarization asymmetries $A_{1}^{N}$ in polarized DIS processes and the single-spin asymmetries $A_{L}^{W^{ \pm}}$in Drell-Yan type processes. Section II is a brief introduction of the conventional calculation about quark distributions by the light-cone quark-spectator-diquark model, and in this sector we get parton distribution functions (PDFs) of both valence and sea quarks as well as polarized PDFs of valence quarks. The polarized PDFs of sea quarks and antiquarks are given in Sec. III from statistical consideration by introducing new parameters: helicity suppression factors $l_{q}(x)$ and $\bar{l}_{q}(x)$ and combined helicity suppression factors $\Delta l_{q}(x)$. The numerical calculations of the nucleon polarization asymmetries $A_{1}^{N}$ and the single-spin asymmetries $A_{L}^{W^{ \pm}}$are presented in Secs. IV and V respectively. We find a significant asymmetry between the quark and antiquark helicity distributions of the nucleon sea. The results show that the quark-antiquark asymmetry of helicity distributions of nucleon sea $q \bar{q}$ pairs, i.e., $\Delta q_{s}(x) \neq \Delta \bar{q}_{s}(x)$, plays an important role for a comprehensive understanding of the nucleon spin content. Finally, we provide a summary.

\section{LIGHT-CONE QUARK-SPECTATOR- DIQUARK MODEL}

The quark-diquark model [52-56] is an effective tool to describe the deep inelastic scattering of leptons on nucleons, with a picture that a single quark is struck by the incident lepton while the remaining part is treated as a quasiparticle of spectator diquark providing the quantum numbers and absorbing nonperturbative effects of all spectating particles. The light-cone SU(6) quark-spectator-diquark model $[10,11,51]$ is actually an updated version by taking into account the relativistic effect of quark transversal motions, with successful applications in investigating hadron structures by calculating relevant physical quantities, such as helicity $[10,16]$ and transversity [11] distributions, form factors [57-60], transverse momentum dependent parton distributions (TMDs) [61-65], generalized parton distributions (GPDs), and Wigner distributions [65]. It is convenient to calculate PDFs and polarized PDFs of valence quarks, and especially it is pointed out in Refs. [8-11] that the relativistic effect due to the Melosh-Wigner rotation [13-15] plays an important role for describing the spinrelated quantities such as the helicity distributions.

As discussions in previous works (see Ref. [10] for example), we can get PDFs and polarized PDFs of valence quarks of the proton from the model:

$$
\begin{aligned}
u_{v}(x) & =\frac{1}{2} a_{S}(x)+\frac{1}{6} a_{V}(x), \\
d_{v}(x) & =\frac{1}{3} a_{V}(x), \\
\Delta u_{v}(x) & =\left[u_{v}(x)-\frac{1}{2} d_{v}(x)\right] W_{S}(x)-\frac{1}{6} d_{v}(x) W_{V}(x), \\
\Delta d_{v}(x) & =-\frac{1}{3} d_{v}(x) W_{V}(x),
\end{aligned}
$$

where $x$ is the light-cone momentum fraction of the quark relative to the nucleon. $a_{D}(x)(D=S$ for scalar diquark and $D=V$ for vector diquark) is the probability when the quark $q$ is struck while the diquark state is $D . a_{D}(x)$ can be written as $a_{D}(x) \propto \int\left[\mathrm{d}^{2} \boldsymbol{k}_{\perp}\right]\left|\varphi_{D}\left(x, \boldsymbol{k}_{\perp}\right)\right|^{2}$ with the normalization form $\int_{0}^{1} a_{D}(x) \mathrm{d} x=3$ and $\boldsymbol{k}_{\perp}$ represents the intrinsic transverse momentum of the quark. $\varphi_{D}\left(x, \boldsymbol{k}_{\perp}\right)$ is the momentum space wave function which we adopt the Brodsky-Huang-Lepage (BHL) prescription [66,67] for light-cone formalism: $\varphi_{D}\left(x, \boldsymbol{k}_{\perp}\right)=A_{D} \exp \left(-\mathcal{M}^{2} / 8 \beta_{D}^{2}\right)$, where $\beta_{D}$ is the harmonic oscillator scale parameter, $A_{D}$ is the normalization constant and $\mathcal{M}$ is the invariant mass: $\mathcal{M}^{2}=\left(m_{q}^{2}+\boldsymbol{k}_{\perp}^{2}\right) / x+\left(m_{D}^{2}+\boldsymbol{k}_{\perp}^{2}\right) /(1-x)$ where $m_{q}$ is the quark mass and $m_{D}$ is the diquark mass. $W_{D}\left(x, \boldsymbol{k}_{\perp}\right)=$ $\left[\left(k^{+}+m_{q}\right)^{2}-\boldsymbol{k}_{\perp}^{2}\right] /\left[\left(k^{+}+m_{q}\right)^{2}+\boldsymbol{k}_{\perp}^{2}\right] \quad$ is the correction factor due to the Melosh-Wigner rotation [8-11] with $k^{+}=x \mathcal{M}$. In this paper, we take the parameter values (see Table I) the same as that given in Set 1 of Ref. [60], which studies the electromagnetic and weak form factors of the ground state octet baryons using the light-cone quarkdiquark model and gives a consistent description of the electroweak properties of the baryons in the low momentum transfer region.

TABLE I. Parameters used in model calculations.

\begin{tabular}{llllll}
\hline \hline Quantity & $m_{q}$ & $m_{S}$ & $m_{V}$ & $\beta_{S}$ & $\beta_{V}$ \\
\hline Value $(\mathrm{MeV})$ & 330 & 600 & 800 & 330 & 330 \\
\hline \hline
\end{tabular}


In order to get a balance between experimental data and model results, we adopt the following parametrization:

$$
\begin{aligned}
u_{v}^{\text {para }}(x) & =u^{\mathrm{CTEQ}}(x)-\bar{u}^{\mathrm{CTEQ}}(x), \\
d_{v}^{\text {para }}(x) & =\frac{d_{v}^{\text {th }}(x)}{u_{v}^{\text {th }}(x)} u_{v}^{\text {para }}(x), \\
\Delta u_{v}^{\text {para }}(x) & =\left[u_{v}^{\text {para }}(x)-\frac{1}{2} d_{v}^{\text {para }}(x)\right] W_{S}(x)-\frac{1}{6} d_{v}^{\text {para }}(x) W_{V}(x), \\
\Delta d_{v}^{\text {para }}(x) & =-\frac{1}{3} d_{v}^{\text {para }}(x) W_{V}(x), \\
u_{s}^{\text {para }}(x) & =\bar{u}_{s}^{\text {para }}(x)=\bar{u}^{\mathrm{CTEQ}}(x), \\
d_{s}^{\text {para }}(x) & =\bar{d}_{s}^{\text {para }}(x)=\bar{d}^{\mathrm{CTEQ}}(x),
\end{aligned}
$$

where the superscript "th" means the pure theoretical results [see Eq. (1)] and "CTEQ" means CTEQ parametrization [68].

\section{LIGHT-FLAVOR SEA (ANTI)QUARK HELICITY DISTRIBUTIONS}

In principle, there is no need to require "the quarkantiquark symmetry of the momentum distributions" for the light-flavor sea quarks. There is no ambiguity for the strange-antistrange asymmetry of the nucleon sea, as have been discussed in the literature. However, the situation becomes complicated for the light-flavor $\mathrm{u}$ and $\mathrm{d}$ cases as there are also valence $u$ and d quarks inside the nucleon, so it is hard to make a definite separation between the sea part and the valence part for the total quark distribution $q(x)=$ $q_{v}(x)+q_{s}(x)$. One convenient definition is to assume "the quark-antiquark symmetry of the momentum distributions", i.e., $q_{s}(x)=\bar{q}(x)$ so that $q_{v}(x)=q(x)-\bar{q}(x)$. Other definition with "the quark-antiquark asymmetry of the momentum distributions" is also possible with theorydependent inputs, but such asymmetry of the momentum distributions is small as from the researches on the strangeantistrange asymmetry of the nucleon sea. However, the quark-antiquark asymmetry might be significant in the situation for the spin-dependent quantities. As a reasonable approximation, we investigate the quark-antiquark asymmetry of helicity distributions in the nucleon sea $q \bar{q}$ pairs while keeping a quark-antiquark symmetry of the momentum distributions as given in Eq. (2).

We assume that the sea helicity distributions are helicitysuppressed by the existence of same-flavor valence helicity distributions with the consideration of the Pauli blocking effect: the existence of a valence quark with certain polarization can cause a suppression of the same-flavor sea quark along the same polarization, and as the nonperturbative sea $q \bar{q}$ pairs are tend to have total spin zero configuration, so the antiquark tends to have the polarization parallel to the valence quark direction. Thus, we assume:
For sea quarks :

$$
\begin{aligned}
& q_{v}^{\uparrow}(x) \rightarrow l_{q}^{\prime}(x) q_{s}^{\downarrow}(x)+\left[1-l_{q}^{\prime}(x)\right] q_{s}^{\uparrow}(x), \\
& q_{v}^{\downarrow}(x) \rightarrow l_{q}^{\prime}(x) q_{s}^{\uparrow}(x)+\left[1-l_{q}^{\prime}(x)\right] q_{s}^{\downarrow}(x) ;
\end{aligned}
$$

For sea antiquarks :

$$
\begin{aligned}
& q_{v}^{\uparrow}(x) \rightarrow \bar{l}_{q}^{\prime}(x) \bar{q}_{s}^{\uparrow}(x)+\left[1-\bar{l}_{q}^{\prime}(x)\right] \bar{q}_{s}^{\downarrow}(x), \\
& q_{v}^{\downarrow}(x) \rightarrow \bar{l}_{q}^{\prime}(x) \bar{q}_{s}^{\downarrow}(x)+\left[1-\bar{l}_{q}^{\prime}(x)\right] \bar{q}_{s}^{\uparrow}(x),
\end{aligned}
$$

where the right arrow $(\rightarrow)$ indicates an impact of valence part distributions on sea part distributions, and $l_{q}^{\prime}(x)$ is the probability of finding a sea quark $q_{s}(q=u, d)$ with polarization antiparallel to the same-flavor valence quark $q_{v}$ at momentum fraction $x$, while $\bar{l}_{q}^{\prime}(x)$ is the probability of finding a sea antiquark $\bar{q}_{s}$ with polarization parallel to the same-flavor valence quark. According to the Pauli blocking effect, we expect $0.5<l_{q}^{\prime}(x) \leq 1$ and $0.5<\bar{l}_{q}^{\prime}(x) \leq 1$. Because $q(x)=q^{\uparrow}(x)+q^{\downarrow}(x)$ and $\Delta q(x)=q^{\uparrow}(x)-q^{\downarrow}(x)$, we can derive the following expressions with Eq. (3):

$$
\begin{aligned}
& \Delta q_{s}(x) \propto \frac{1}{1-2 l_{q}^{\prime}(x)} \frac{\Delta q_{v}(x)}{q_{v}(x)} q_{s}(x), \\
& \Delta \bar{q}_{s}(x) \propto-\frac{1}{1-2 \bar{l}_{q}^{\prime}(x)} \frac{\Delta q_{v}(x)}{q_{v}(x)} \bar{q}_{s}(x) .
\end{aligned}
$$

For convenience, we assume:

$$
\begin{aligned}
& \Delta q_{s}(x)=l_{q}(x) \frac{\Delta q_{v}(x)}{q_{v}(x)} q_{s}(x), \\
& \Delta \bar{q}_{s}(x)=-\bar{l}_{q}(x) \frac{\Delta q_{v}(x)}{q_{v}(x)} \bar{q}_{s}(x),
\end{aligned}
$$

where $l_{q}(x)$ and $\bar{l}_{q}(x)$, which can be called helicity suppression factors, reflect the helicity suppression effects of q-flavor valence quark on the same-flavor sea quark and antiquark respectively. From Eq. (4) and the Pauli blocking effect, we expect $l_{q}(x) \leq 0$ and $\bar{l}_{q}(x) \leq 0$, which are confirmed by following numerical calculations (see Table IV). It is obvious that $\left|l_{q}(x) \frac{\Delta q_{v}(x)}{q_{v}(x)}\right| \leq 1$ and $\left|\bar{l}_{q}(x) \frac{\Delta q_{v}(x)}{q_{v}(x)}\right| \leq 1$.

Two schemes are used to solve $l_{q}(x)$ and $\bar{l}_{q}(x)$ :

(1) $\left\{\begin{array}{l}l_{q}(x)=l_{q}, \\ \bar{l}_{q}(x)=\bar{l}_{q},\end{array}\right.$

(2) $\left\{\begin{array}{l}l_{q}(x)=l_{q} \times \alpha(x), \\ I_{q}(x)=\bar{l}_{q} \times \alpha(x),\end{array}\right.$ where $\alpha(x)$ is an adjusting function with the characteristic features: the function tends to 1 when $x$ is not so small, and to 0 when $x$ is very small. We recommend $\alpha(x)=\exp \left(-c x^{-1}\right)$ (see Fig. 1) with $c=0.015$ (which is adjustable) as an option. The reason for this choice is based on the consideration that the impact of valence part on sea part should be big when $x$ is not so small and little when $x$ is very small. 


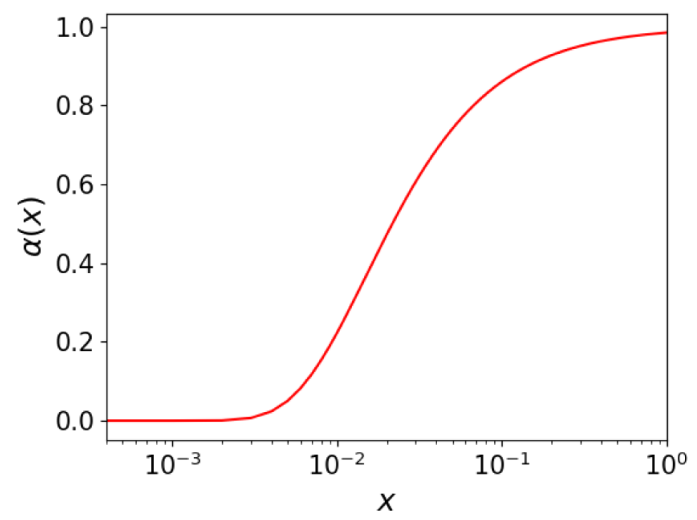

FIG. 1. The adjusting function $\alpha(x)$ as a function of the momentum fraction $x$. The function tends to 1 when $x$ is not so small, and to 0 when $x$ is very small.

Furthermore, because the quark-antiquark symmetry of the momentum distributions [i.e., $q_{s}(x)=\bar{q}_{s}(x)$ ] is assumed, we can define:

$$
\begin{aligned}
\Delta q_{s+\bar{s}}= & \Delta q_{s}(x)+\Delta \bar{q}_{s}(x)=\Delta l_{q}(x) \frac{\Delta q_{v}(x)}{q_{v}(x)} q_{s}(x), \\
& \text { with } \quad \Delta l_{q}(x)=l_{q}(x)-\bar{l}_{q}(x),
\end{aligned}
$$

where $\Delta l_{q}(x)$, which can be called combined helicity suppression factor of quark $q$, represents the combined helicity suppression effect.

\section{NUCLEON POLARIZATION ASYMMETRIES $A_{1}^{N}$ IN POLARIZED DIS PROCESSES}

With the PDFs and polarized PDFs derived above and due to isospin symmetry between proton and neutron, we can get spin-independent structure functions $F_{2}^{N}(x)$ $(N=p, n)$ :

$$
\begin{aligned}
& F_{2}^{p}(x)=x\left\{\frac{4}{9}[u(x)+\bar{u}(x)]+\frac{1}{9}[d(x)+\bar{d}(x)]\right\}, \\
& F_{2}^{n}(x)=x\left\{\frac{1}{9}[u(x)+\bar{u}(x)]+\frac{4}{9}[d(x)+\bar{d}(x)]\right\},
\end{aligned}
$$

where $q(x)=q_{v}(x)+q_{s}(x), \quad \bar{q}(x)=\bar{q}_{s}(x)$ and spindependent structure functions $g_{1}^{N}(x)(N=p, n)$ :

$$
\begin{aligned}
g_{1}^{p}(x)= & \frac{1}{2}\left\{\frac{4}{9}\left[\Delta u_{v}(x)+\Delta u_{s+\bar{s}}(x)\right]\right. \\
& \left.+\frac{1}{9}\left[\Delta d_{v}(x)+\Delta d_{s+\bar{s}}(x)\right]\right\}, \\
g_{1}^{n}(x)= & \frac{1}{2}\left\{\frac{1}{9}\left[\Delta u_{v}(x)+\Delta u_{s+\bar{s}}(x)\right]\right. \\
& \left.+\frac{4}{9}\left[\Delta d_{v}(x)+\Delta d_{s+\bar{s}}(x)\right]\right\},
\end{aligned}
$$

where $\Delta q_{s+\bar{s}}$ is defined in Eq. (6). The nucleon polarization asymmetries $A_{1}^{N}(N=p, n)$ are directly measured in experiments and expressed as $A_{1}^{N}(x)=2 x g_{1}^{N}(x) / F_{2}^{N}(x)$.

In the nucleon sea, the polarization asymmetries $A_{1}^{N}$ are only sensitive to the total helicity distribution functions $\left(\Delta q_{s+\bar{s}}\right)$ so that we only need to fit the combined helicity suppression factors $\Delta l_{q}(x)(q=u, d)$. We get $\Delta l_{q}(x)$ $(q=u, d)$ by fitting experimental data of proton polarization asymmetry $A_{1}^{p}$ at E130 [49], EMC [1], SMC [18], E143 [19] and neutron polarization asymmetry $A_{1}^{n}$ at E142 [20], E154 [50]. The fitting method is:

$$
\chi^{2}=\sum_{i}\left(\mathcal{O}_{i}^{\exp }-\mathcal{O}_{i}^{\text {th }}\right)^{2}\left(\sigma_{i}^{2}\right)^{-1}
$$

where $\mathcal{O}_{i}^{\text {exp }}$ and $\mathcal{O}_{i}^{\text {th }}$ are experimental and theoretical values at the $i$ 's data respectively, and $\sigma_{i}$ is $i$ 's experimental uncertainty.

The fitting results of combined helicity suppression factors $\left(\Delta l_{u}\right.$ and $\left.\Delta l_{d}\right)$ are exhibited in Table II. Because $\Delta u_{v}>0$ and $\Delta d_{v}<0$, we come to the conclusion that $\Delta u_{s+\bar{s}}<0$ and $\Delta d_{s+\bar{s}}<0$. The calculated nucleon polarization asymmetries of proton $A_{1}^{p}$ and neutron $A_{1}^{n}$ as functions of $x$ are shown in Fig. 2 and compared with experimental data. By considering sea helicity distributions, the theoretical calculation results of $A_{1}^{N}$ are better consistent with the experimental data. Two schemes are adopted and a noticeable deviation appears at small $x$. The fitting results (solid curves) of Scheme 2 are in good agreement with the experimental data, while the results of Scheme 1 deviate from the experimental data at small $x$.

In addition, because the spin dependent structure functions $g_{1}^{N}(x)$ are related to the net quark helicity in nucleon, the first moments $\Gamma_{1}^{N}=\int_{0}^{1} g_{1}^{N}(x) \mathrm{d} x$ are checked. In Table III, we give the calculation results without the contribution of the sea part and the results with the contribution of the sea part of two schemes. By comparing with results of COMPASS [25], we notice that the theoretical results of Scheme 1 are in agreement with experimental results when $x$ is not so small $[x>0.0025(0.004)]$, but deviate from experimental results when $x$ is very small $[x<0.0025(0.004)]$. This kind of behavior is consistent with that analyzed above when discussing the nucleon polarization asymmetries. The behavior of Scheme 1 may be caused by the changeless influence of valence part on sea part and this hypothesis of

TABLE II. Fitting results of combined helicity suppression factors $\left(\Delta l_{u}\right.$ and $\left.\Delta l_{d}\right)$.

\begin{tabular}{lcc}
\hline \hline Scheme & $\Delta l_{u}$ & $\Delta l_{d}$ \\
\hline 1 & -0.421 & 0.586 \\
2 & -0.713 & 0.885 \\
\hline \hline
\end{tabular}




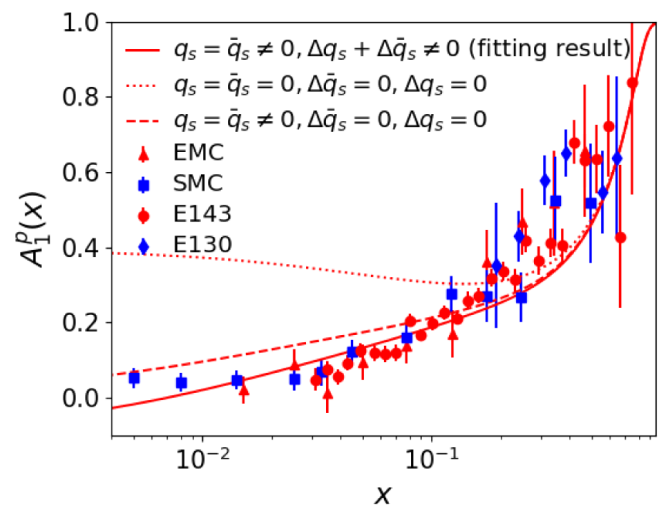

(a) Scheme 1: proton spin asymmetry $A_{1}^{p}(x)$.

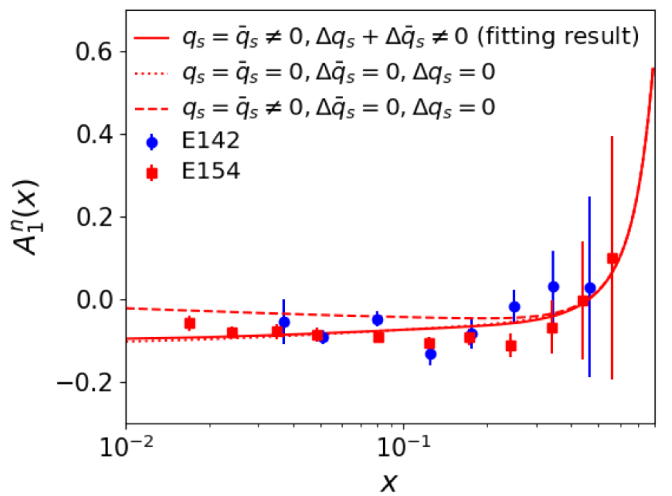

(c) Scheme 1: neutron spin asymmetry $A_{1}^{n}(x)$.

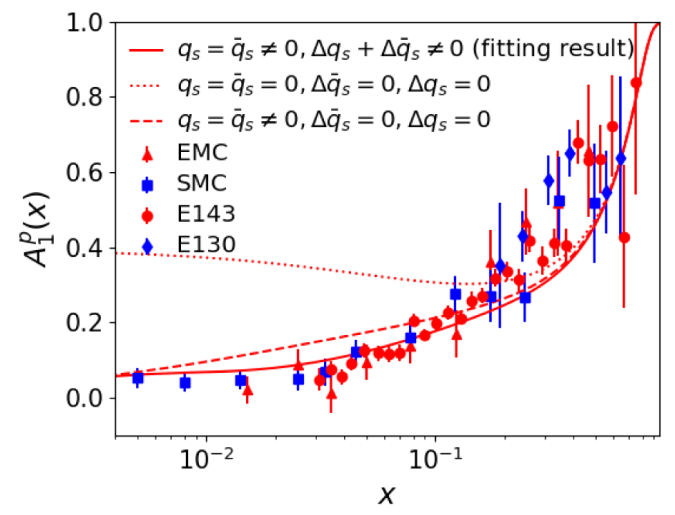

(b) Scheme 2: proton spin asymmetry $A_{1}^{p}(x)$.

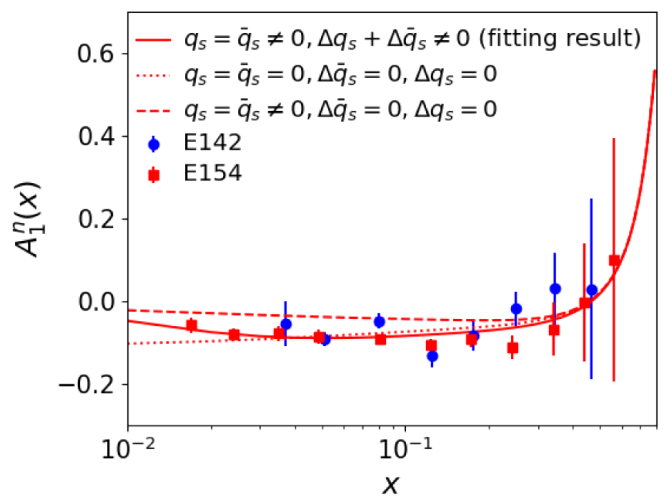

(d) Scheme 2: neutron spin asymmetry $A_{1}^{n}(x)$.

FIG. 2. The nucleon polarization asymmetries $A_{1}^{N}(x)$ : (a) and (b) for the proton with data from SMC [18], E143 [19], E130 [49], EMC [1]; (c) and (d) for the neutron with data from E142 [20], E154 [50]. The dotted curves are the results without considering the sea contributions. The dashed curves take into account the contributions of unpolarized distributions of sea part but not the polarized ones. The solid curves consider both unpolarized and polarized sea contributions and are fitting results.

the changeless influence is perhaps inadequate because of the considerable parton distribution of sea quarks compared to the valence part at small $x$. The situation is improved when it comes to Scheme 2, which is exactly the one that is arranged with a variable valence influence. We notice that the results of Scheme 2 are in good agreement with COMPASS results [25] in each interval of $x$.

TABLE III. The results of $\Gamma_{1}^{p}, \Gamma_{1}^{n}$, and $\Gamma_{1}^{N}\left(\Gamma_{1}^{N}=\frac{1}{2}\left(\Gamma_{1}^{p}+\Gamma_{1}^{n}\right)\right)$.

\begin{tabular}{|c|c|c|c|c|c|}
\hline & $x$ range & Scheme 1 & Scheme 2 & $\begin{array}{l}\text { Without } \\
\text { sea part }\end{array}$ & $\begin{array}{cc}t & \text { COMPASS } \\
t & {[25]}\end{array}$ \\
\hline \multirow[t]{4}{*}{$\overline{\Gamma_{1}^{p}}$} & $0-0.0025$ & -0.031 & 0.012 & 0.012 & 0.002 \\
\hline & $0.0025-0.7$ & 0.115 & 0.134 & 0.159 & $0.134 \pm 0.003$ \\
\hline & $0.7-1.0$ & 0.001 & 0.001 & 0.001 & 0.003 \\
\hline & $0-1$ & 0.086 & 0.147 & 0.172 & $0.139 \pm 0.006$ \\
\hline \multirow[t]{4}{*}{$\Gamma_{1}^{N}$} & $0-0.004$ & -0.042 & 0.006 & 0.006 & 0.000 \\
\hline & $0.004-0.7$ & 0.029 & 0.041 & 0.065 & $0.047 \pm 0.003$ \\
\hline & $0.7-1$ & 0.001 & & 0.001 & 0.001 \\
\hline & $0-1$ & -0.012 & 0.048 & 0.073 & $0.049 \pm 0.003$ \\
\hline$\Gamma_{1}^{n}$ & $0-1$ & -0.110 & -0.052 & -0.027 & $-0.041 \pm 0.006$ \\
\hline
\end{tabular}

We modify the nucleon spin asymmetries by considering the helicity distributions of sea quarks and antiquarks. Nevertheless, we only get the combined helicity suppression factors $\left[\Delta l_{u}(x)\right.$ and $\left.\Delta l_{d}(x)\right]$, but having no idea about the single helicity suppression effects of sea quarks as well as antiquarks. We will check these effects in the following section by calculating single-spin asymmetries $A_{L}^{W^{ \pm}}$in Drell-Yan type processes.

\section{SINGLE-SPIN ASYMMETRIES $A_{L}^{W^{ \pm}}$IN $\vec{p}+p$ COLLISIONS}

Due to the pure V-A structure of weak interaction vertex $W q \bar{q}^{\prime}$ and because of quark helicity conservation at the vertex, the single-spin asymmetries $A_{L}^{W^{+}}$is sensitive to $\Delta \bar{d}_{s}(x)$ and $\Delta u_{s}(x)$, while $A_{L}^{W^{-}}$to $\Delta \bar{u}_{s}(x)$ and $\Delta d_{s}(x)$, which allow us to explore the helicity suppression effects of sea quarks and antiquarks individually.

At leading order (LO), the single-spin asymmetry $A_{L}^{W^{+}}$ with midprocess $u \bar{d} \rightarrow W^{+}$and $A_{L}^{W^{-}}$with midprocess $d \bar{u} \rightarrow W^{-}$can be expressed as: 
TABLE IV. Fitting results of $\bar{l}_{u}$ and $\bar{l}_{d}$ and calculated results of $l_{u}$ and $l_{d}$.

\begin{tabular}{lcccc}
\hline \hline Scheme & $\bar{l}_{u}$ & $\bar{l}_{d}$ & $l_{u}$ & $l_{d}$ \\
\hline 1 & -0.751 & -1.347 & -1.172 & -0.761 \\
2 & -0.836 & -1.658 & -1.549 & -0.773 \\
\hline \hline
\end{tabular}

$$
\begin{aligned}
& A_{L}^{W^{+}}=\frac{-\Delta u\left(x_{1}\right) \bar{d}\left(x_{2}\right)+\Delta \bar{d}\left(x_{1}\right) u\left(x_{2}\right)}{u\left(x_{1}\right) \bar{d}\left(x_{2}\right)+\bar{d}\left(x_{1}\right) u\left(x_{2}\right)}, \\
& A_{L}^{W^{-}}=\frac{-\Delta d\left(x_{1}\right) \bar{u}\left(x_{2}\right)+\Delta \bar{u}\left(x_{1}\right) d\left(x_{2}\right)}{d\left(x_{1}\right) \bar{u}\left(x_{2}\right)+\bar{u}\left(x_{1}\right) d\left(x_{2}\right)},
\end{aligned}
$$

with

$$
\begin{array}{rlrl}
q(x) & =q_{v}(x)+q_{s}(x), & \bar{q}(x)=\bar{q}_{s}(x), \\
\Delta q(x) & =\Delta q_{v}(x)+\Delta q_{s}(x), \quad \Delta \bar{q}(x)=\Delta \bar{q}_{s}(x),
\end{array}
$$

where $q=u, d$.

With the combination of Eq. (6) and fitting results of $\Delta l_{u}(x)$ and $\Delta l_{d}(x)$ (see Table II) together, a simplification can be made by fitting two parameters $\left[\bar{l}_{u}(x)\right.$ and $\left.\bar{l}_{d}(x)\right]$

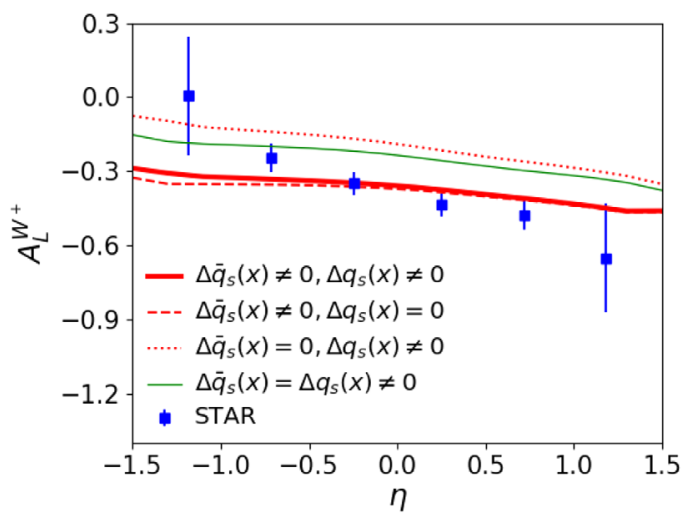

(a) Scheme 1: single-spin asymmetries $A_{L}^{W^{+}}$.

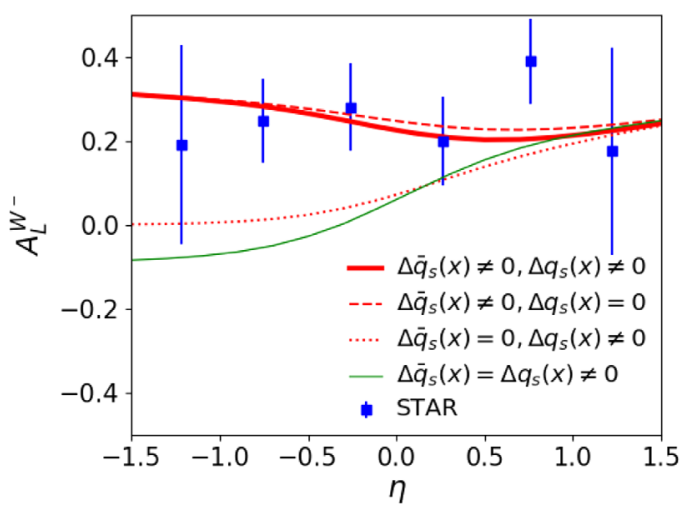

(c) Scheme 1: single-spin asymmetries $A_{L}^{W^{-}}$. instead of four with the method declared in Eq. (9). We get $\bar{l}_{u}$ and $\bar{l}_{d}$ by fitting experimental data of single-spin asymmetries $A_{L}^{W^{ \pm}}$at RHIC [23]. The fitting results of $\bar{l}_{u}$ and $\bar{l}_{d}$ and calculated results of $l_{u}$ and $l_{d}$ are exhibited in Table IV. The calculated single-spin asymmetries $A_{L}^{W^{ \pm}}$as functions of $x$ are shown in Fig. 3 and compared with experimental data. More calculation details can be found in Refs. [21,69].

In Fig. 3, the curves are calculated with the results of combined suppression factors $\left[\Delta l_{u}(x)\right.$ and $\left.\Delta l_{d}(x)\right]$ obtained in Sec. IV. The thin-solid (green) curves are directly calculated by adopting a quark-antiquark symmetry of helicity distributions [i.e., $\Delta q_{s}(x)=\Delta \bar{q}_{s}(x)$ ], while the thick-solid, dotted and dashed (red) curves are calculated by a second fitting without a preassumption of the quarkantiquark symmetry of helicity distributions, and the fitting results show a bulk of quark-antiquark asymmetry [i.e., $\left.\Delta q_{s}(x) \neq \Delta \bar{q}_{s}(x)\right]$. The bad performances of the thin-solid (green) curves accord with the results in Ref. [21], which show that it is difficult to give a simultaneous description of both the experimental data from DIS processes and that from $W^{ \pm}$productions with a quark-antiquark symmetry of

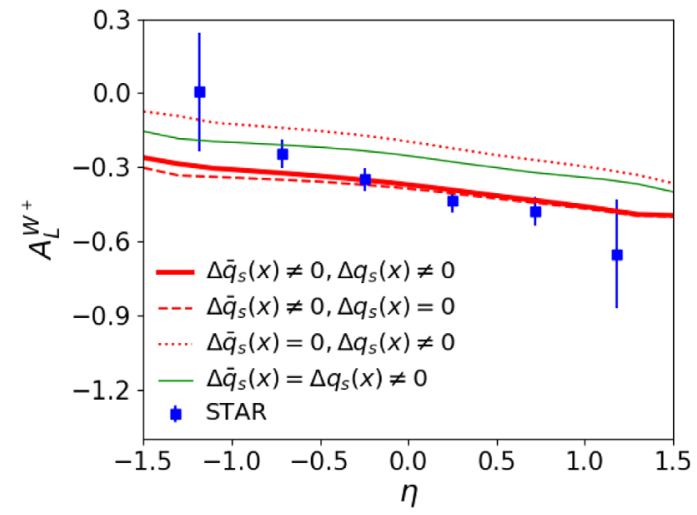

(b) Scheme 2: single-spin asymmetries $A_{L}^{W^{+}}$.

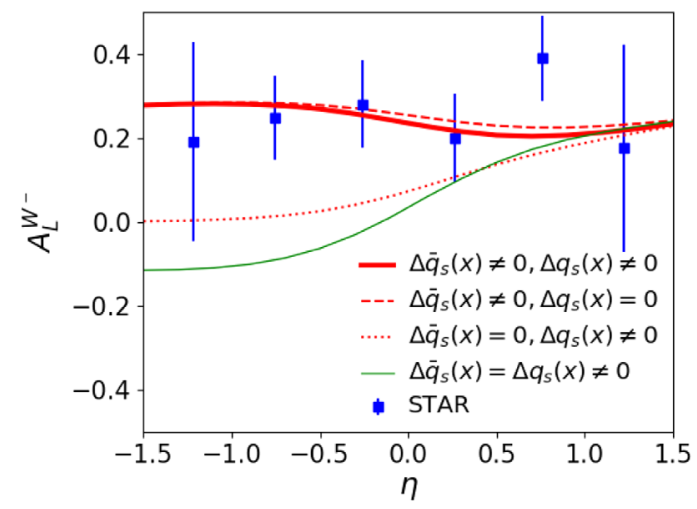

(d) Scheme 2: single-spin asymmetries $A_{L}^{W^{-}}$.

FIG. 3. The single-spin asymmetries $A_{L}^{W^{ \pm}}$as a function of the lepton pseudorapidity $\eta$. The data are from [23]. The thick (red) solid curves are the fitting results, while the thin (green) solid curves adopt the assumption $\Delta q_{s}(x)=\Delta \bar{q}_{s}(x)$ obtained by $\Delta l(x)$. Dotted curves only consider the sea quark helicity distributions, and dashed curves only consider the sea antiquark helicity distributions. 
helicity distributions. However, after taking into account the quark-antiquark asymmetry of helicity distribution of nucleon sea $q \bar{q}$ pairs, the thick-solid (red) curves are consistent with the experimental data. The thick-solid curves consider the helicity contributions of both the quarks and antiquarks in nucleon sea, while the dotted curves and dashed curves only consider the helicity contributions of sea quarks and antiquarks respectively. The curves show that the contribution of helicity distributions of sea antiquarks are much bigger than that of sea quarks. Two different schemes make little difference and the Scheme 2 is a little better than Scheme 1. From the results shown in Table IV, the helicity suppression factors $\left(\bar{l}_{u}, \bar{l}_{d}, l_{u}\right.$ and $\left.l_{d}\right)$ are all negative, which is expected as discussed in Sec. III from statistical consideration of the Pauli blocking effect.

Figure 4 shows the results of $\Delta q(x) / q(x)$ and $\Delta \bar{q}(x) / \bar{q}(x)$ as functions of the momentum fraction $x$. Figure 5 shows the results of $x \Delta q(x)$ and $x \Delta \bar{q}(x)$ as functions of the momentum fraction $x$. The behaviors about $x \Delta q(x)$ and $x \Delta \bar{q}(x)$ are consistent with the statistical consideration [70-72], the phenomenological analysis [21] as well as the parametrized results in Refs. [73,74], which study the asymmetries $A_{1}^{N}, A_{1}^{p, \pi^{ \pm}}$and $A_{1}^{p, K^{ \pm}}$in inclusive and semi-inclusive polarized DIS processes. The results also indicate a significant flavor asymmetry of the antiquark helicity distributions as predicted from some theoretical considerations [75-79].

The model calculation results of quantities $\Delta q$ $\left(\Delta q=\int_{0}^{1} q(x) \mathrm{d} x\right)$ are shown in Table V. The results of Scheme 1 and Scheme 2 are very different, especially when calculating $\Delta d$ and $\Delta \Sigma$ (for example, the result of $\Delta \Sigma$ of Scheme 2 is positive, while that of Scheme 1 is negative). The difference of the results comes from the different impact of valence part on sea part of these two schemes as elaborated in Sec. III. In Scheme 1, the impact of valence part helicity distributions on sea part helicity distributions is changeless in the range of the entire independent variable $x$, while in Scheme 2, the impact is variable with $x$. Compared with parametrized results $\left(\Delta u^{+}, \Delta d^{+}\right.$, and $\left.\Delta \Sigma\right)$ of NNPDFpl11.1 [80] and DSSV08 [81], Scheme 2 performs much better than Scheme 1 as discussed in Sec. IV, and the results of Scheme 2 are in pretty good agreement with these two parametrized results.

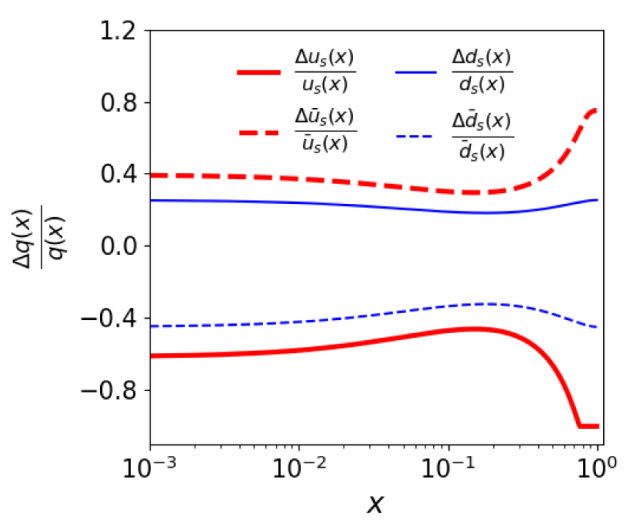

(a) Scheme 1: $\frac{\Delta q(x)}{q(x)}$.

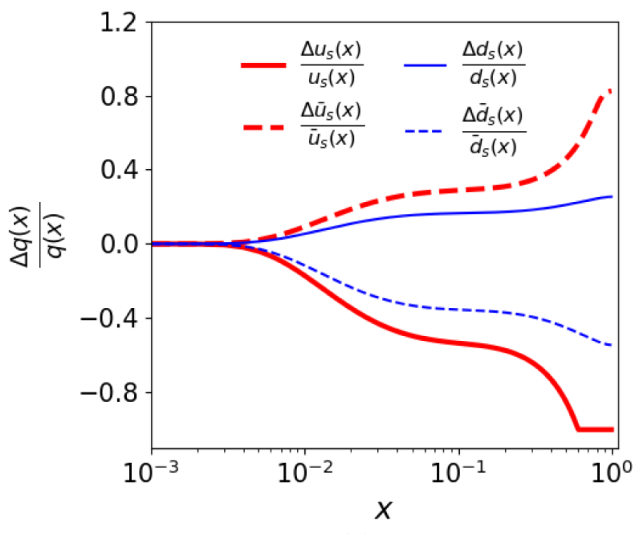

(b) Scheme 2: $\frac{\Delta q(x)}{q(x)}$.

FIG. 4. $\frac{\Delta q(x)}{q(x)}$ as functions of the momentum fraction $x$ in two schemes.

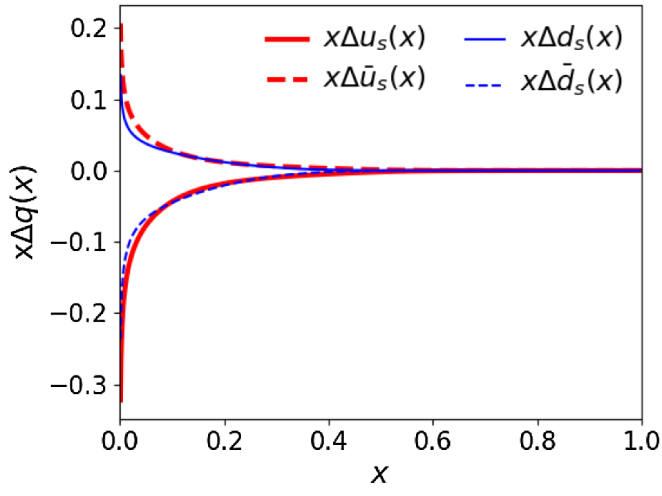

(a) Scheme 1: $x \Delta q(x)$.

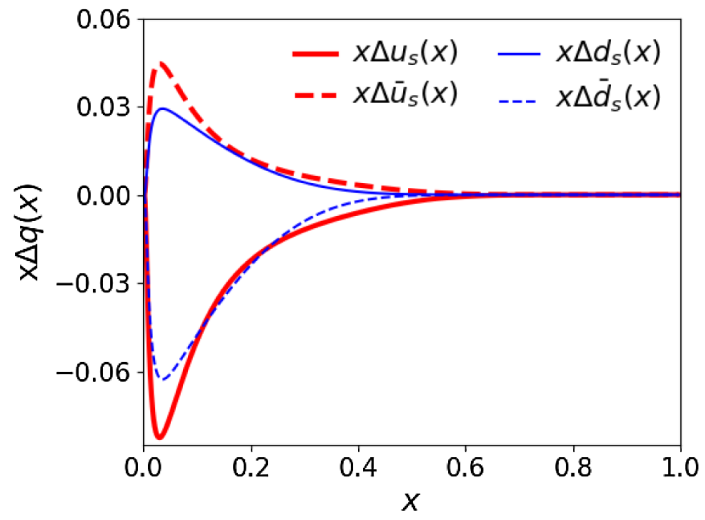

(b) Scheme 2: $x \Delta q(x)$.

FIG. 5. $x \Delta q(x)$ as functions of the momentum fraction $x$ in two schemes. 
TABLE V. $\Delta q$, quantities from model calculations.

\begin{tabular}{lcccr}
\hline \hline Scheme & $\Delta u_{v}$ & $\Delta u_{s}$ & $\Delta \bar{u}_{s}$ & $\Delta u$ \\
\hline 1 & 0.827 & -0.808 & 0.518 & 0.019 \\
2 & 0.827 & -0.221 & 0.120 & 0.606 \\
\hline \hline Scheme & $\Delta d_{v}$ & $\Delta d_{s}$ & $\Delta \bar{d}_{s}$ & $\Delta d$ \\
\hline 1 & -0.329 & 0.353 & -0.624 & 0.024 \\
2 & -0.329 & 0.083 & -0.177 & -0.247 \\
\hline
\end{tabular}

\begin{tabular}{lccc}
\hline \hline Scheme & $\Delta u^{+}$ & $\Delta d^{+}$ & $\Delta \Sigma$ \\
\hline 1 & 0.537 & -0.601 & -0.064 \\
2 & 0.725 & -0.424 & 0.302 \\
NNPDFpl11.1 [80] & $0.76 \pm 0.04$ & $-0.41 \pm 0.04$ & $0.25 \pm 0.10$ \\
DSSV08 [81] & $0.793_{-0.034}^{+0.028}(+0.020)$ & $-0.416_{-0.025}^{+0.035}(-0.042)$ & $0.366_{-0.062}^{+0.042}(+0.123)$ \\
\hline \hline
\end{tabular}

Back to the "proton spin crisis," recent studies [3-5] reported about $30 \%$ of the proton spin coming from quark spins. With the discussion about spin and dynamics given in Introduction, we can understand the "spin crisis" by recognizing the quantity $\Delta \Sigma\left(\Delta \Sigma=\Delta u^{+}+\Delta d^{+}\right.$with $\left.\Delta q^{+}=\Delta q_{v}+\Delta q_{s}+\Delta \bar{q}_{s}\right)$ as the sum of quark helicities in light-form dynamics rather than the vector sum of spins carried by quarks and antiquarks in the proton rest frame. Therefore, the quantity $\Delta \Sigma$, representing the light-cone spin sum of quarks and antiquarks inside the proton, plays an important role in studying the nucleon spin structure. As shown in Table V, the theoretical calculation results of $\Delta \Sigma$ of Scheme 2 is 0.302 , which just falls on the parametrized results: $0.25 \pm 0.10$ from NNPDFpl11.1 [80] and $0.366_{-0.062}^{+0.042}(+0.123)$ from DSSV08 [81]. Thus Scheme 2 provides a reasonable scenario to calculate sea quark and antiquark helicity distributions from statistical consideration. The results indicate that the light-cone SU(6) quarkspectator-diquark model, together with the relativistic effect due to quark transversal motions and the quark-antiquark asymmetry of helicity distributions of the nucleon sea with phenomenologically based models and parameters, can provide good descriptions for the physical quantities related to the "proton spin crisis."

\section{SUMMARY}

In summary, we study the helicity distributions of light flavor $q \bar{q}$ pairs in the nucleon sea. We investigate the contributions of sea quark and antiquark helicity distributions to nucleon polarization asymmetries $A_{1}^{N}$ in DIS processes and single-spin asymmetries $A_{L}^{W^{ \pm}}$in Drell-Yan type processes. The results show that there is a significant asymmetry between quark and antiquark helicity distributions of the nucleon sea. We compare the model results of $\Gamma_{1}^{N}, x \Delta q(x)$ and $\Delta q(N=p, n)$ with experimental [25], parametrized [73,74,80,81], statistical [70-72], and phenomenological [21] results, and they conform well. From statistical consideration, we assume that the sea helicity distributions are helicity-suppressed by the existence of same-flavor valence helicity distributions. With this statistical assumption, we give the method of calculating helicity distributions of sea quarks and antiquarks by introducing new parameters: helicity suppression factors $l_{q}(x)$ and $\bar{l}_{q}(x)$ and combined helicity suppression factors $\Delta l_{q}(x)$. Two schemes are put forward to help calculations. The good performance of the second scheme show that because of the relative quantitative relation between valence quarks and sea (anti)quarks, the influence of valence part helicity distributions on sea part helicity distributions is variable with the momentum fraction $x$ : the influence is small when $x$ is very small, while big when $x$ is not so small. It is shown that the quark-antiquark asymmetry of helicity distributions of the nucleon sea is important for a simultaneous description of the nucleon polarization asymmetries $A_{1}^{N}$ in inclusive DIS processes and the single-spin asymmetries $A_{L}^{W^{ \pm}}$in Drell-Yan type processes. Moreover, the model result of the quantity $\Delta \Sigma$ (which is usually stated as the quark spin content of the proton) just falls on the parametrized results. Therefore the quark-antiquark asymmetry [i.e., $\Delta q_{s}(x) \neq \Delta \bar{q}_{s}(x)$ ] plays an important role for a comprehensive understanding of the nucleon spin structure.

\section{ACKNOWLEDGMENTS}

This work is supported by National Natural Science Foundation of China (Grant No. 11475006). 
[1] J. Ashman et al. (European Muon Collaboration), A measurement of the spin asymmetry and determination of the structure function $g_{1}$ in deep inelastic muon-proton scattering, Phys. Lett. B 206, 364 (1988).

[2] J. Ashman et al. (European Muon Collaboration), An investigation of the spin structure of the proton in deep inelastic scattering of polarized muons on polarized protons, Nucl. Phys. B328, 1 (1989).

[3] E. S. Ageev et al. (COMPASS Collaboration), Measurement of the spin structure of the deuteron in the DIS region, Phys. Lett. B 612, 154 (2005).

[4] V. Y. Alexakhin et al. (COMPASS Collaboration), The deuteron spin-dependent structure function $g_{1}^{d}$ and its first moment, Phys. Lett. B 647, 8 (2007).

[5] A. Airapetian et al. (HERMES Collaboration), Precise determination of the spin structure function $g_{1}$ of the proton, deuteron and neutron, Phys. Rev. D 75, 012007 (2007).

[6] M. Gell-Mann, A schematic model of baryons and mesons, Phys. Lett. 8, 214 (1964).

[7] G. Zweig, An SU(3) model for strong interaction symmetry and its breaking. Version 1, CERN Report No. 8182/TH.401, 1964; An SU(3) model for strong interaction symmetry and its breaking. Version 2, CERN Report No. 8419/TH.412, 1964.

[8] B.-Q. Ma, Melosh rotation: Source for the proton's missing spin, J. Phys. G 17, L53 (1991).

[9] B.-Q. Ma, The proton spin and the Wigner rotation, Z. Phys. C 58, 479 (1993).

[10] B.-Q. Ma, The $x$-dependent helicity distributions for valence quarks in nucleons, Phys. Lett. B 375, 320 (1996).

[11] B.-Q. Ma, I. Schmidt, and J. Soffer, The quark spin distributions of the nucleon, Phys. Lett. B 441, 461 (1998).

[12] P. A. M. Dirac, Forms of relativistic dynamics, Rev. Mod. Phys. 21, 392 (1949).

[13] E. P. Wigner, On unitary representations of the inhomogeneous Lorentz group, Ann. Math. 40, 149 (1939); Nucl. Phys. B, Proc. Suppl. 6, 9 (1989).

[14] H. J. Melosh, Quarks: Currents and constituents, Phys. Rev. D 9, 1095 (1974).

[15] F. Buccella, C. A. Savoy, and P. Sorba, Current quarks, constituent quarks and the Poincare group, Lett. Nuovo Cimento Soc. Ital. Fis. 10, 455 (1974).

[16] X. Chen, Y. Mao, and B.-Q. Ma, Parity-violating asymmetry of $W$ bosons produced in $p-p$ collisions, Nucl. Phys. A759, 188 (2005).

[17] B. Adeva et al. (Spin Muon Collaboration), Measurement of the spin dependent structure function $g_{1}(x)$ of the deuteron, Phys. Lett. B 302, 533 (1993).

[18] D. Adams et al. (Spin Muon (SMC) Collaboration), Measurement of the spin dependent structure function $g_{1}(x)$ of the proton, Phys. Lett. B 329, 399 (1994); Erratum, Phys. Lett. B339, 332(E) (1994).

[19] K. Abe et al. (E143 Collaboration), Precision Measurement of the Proton Spin Structure Function $g_{1}^{p}$, Phys. Rev. Lett. 74, 346 (1995).

[20] P. L. Anthony et al. (E142 Collaboration), Determination of the Neutron Spin Structure Function, Phys. Rev. Lett. 71, 959 (1993).

[21] F. Tian, C. Gong, and B.-Q. Ma, Effect of sea quarks on the single-spin asymmetries $A_{L}^{W^{ \pm}}$in polarized $p p$ collisions at RHIC, Nucl. Phys. A961, 154 (2017).
[22] F. Tian, C. Gong, and B.-Q. Ma, Effect of sea quarks on single-spin asymmetries $A_{N}^{W^{ \pm}}$in transversely polarized pp collisions at RHIC, Nucl. Phys. A968, 379 (2017).

[23] L. Adamczyk et al. (STAR Collaboration), Measurement of Longitudinal Spin Asymmetries for Weak Boson Production in Polarized Proton-Proton Collisions at RHIC, Phys. Rev. Lett. 113, 072301 (2014).

[24] L. Adamczyk et al. (STAR Collaboration), Measurement of the Transverse Single-Spin Asymmetry in $p^{\uparrow}+p \rightarrow$ $W^{ \pm} / Z^{0}$ at RHIC, Phys. Rev. Lett. 116, 132301 (2016).

[25] C. Adolph et al. (COMPASS Collaboration), The spin structure function $g_{1}^{\mathrm{p}}$ of the proton and a test of the Bjorken sum rule, Phys. Lett. B 753, 18 (2016).

[26] S. J. Brodsky and B.-Q. Ma, The quark-antiquark asymmetry of the nucleon sea, Phys. Lett. B 381, 317 (1996).

[27] A. I. Signal and A. W. Thomas, Possible strength of the nonperturbative strange sea of the nucleon, Phys. Lett. B 191, 205 (1987).

[28] M. Burkardt and B. J. Warr, Chiral symmetry and the charge asymmetry of the $s \bar{s}$ distribution in the proton, Phys. Rev. D 45, 958 (1992).

[29] A. Szczurek, H. Holtmann, and J. Speth, Four-quark and six-quark components in the pion light front wave function and nonperturbative sea in the pion, Nucl. Phys. A605, 496 (1996).

[30] B.-Q. Ma, Strange magnetic moment and isospin symmetry breaking, Phys. Lett. B 408, 387 (1997).

[31] Y. Ding and B.-Q. Ma, Contribution of asymmetric strangeantistrange sea to the Paschos-Wolfenstein relation, Phys. Lett. B 590, 216 (2004).

[32] Y. Ding, R. G. Xu, and B.-Q. Ma, Effect of asymmetric strange-antistrange sea to the NuTeV anomaly, Phys. Lett. B 607, 101 (2005).

[33] M. Wakamatsu, On the NuTeV anomaly and the asymmetry of the strange sea in the nucleon, Phys. Rev. D 71, 057504 (2005).

[34] Y. Ding, R. G. Xu, and B.-Q. Ma, Nucleon sea in the effective chiral quark model, Phys. Rev. D 71, 094014 (2005).

[35] J. Alwall and G. Ingelman, Quark asymmetries in the proton from a model for parton densities, Phys. Rev. D 71, 094015 (2005).

[36] M. Wakamatsu, Flavor structure of the unpolarized and longitudinally-polarized sea-quark distributions in the nucleon, Phys. Rev. D 90, 034005 (2014).

[37] V. Barone, C. Pascaud, and F. Zomer, A new global analysis of deep inelastic scattering data, Eur. Phys. J. C 12, 243 (2000).

[38] C. Bourrely, J. Soffer, and F. Buccella, Strangeness asymmetry of the nucleon in the statistical parton model, Phys. Lett. B 648, 39 (2007).

[39] A. Vega, I. Schmidt, T. Gutsche, and V. E. Lyubovitskij, Nonperturbative contribution to the strange-antistrange asymmetry of the nucleon sea, Phys. Rev. D 93, 056001 (2016).

[40] M. Diehl, T. Feldmann, and P. Kroll, Form factors and other measures of strangeness in the nucleon, Phys. Rev. D 77, 033006 (2008).

[41] T. J. Hobbs, M. Alberg, and G. A. Miller, Constraining nucleon strangeness, Phys. Rev. C 91, 035205 (2015). 
[42] P. Gao and B.-Q. Ma, Probing nucleon strange asymmetry from charm production in neutrino deep inelastic scattering, Eur. Phys. J. C 44, 63 (2005).

[43] G. Hao, L. Li, and C.F. Qiao, $D_{s}^{+}-D_{s}^{-}$asymmetry in photoproduction, Phys. Lett. B 621, 139 (2005).

[44] P. Gao and B.-Q. Ma, The leading particle effect from light quark fragmentation in charm hadroproduction, Eur. Phys. J. C 50, 603 (2007).

[45] P. Gao and B.-Q. Ma, Influence of heavy quark recombination on the nucleon strangeness asymmetry, Phys. Rev. D 77, 054002 (2008).

[46] S. S. Zhou, Y. Chen, Z. T. Liang, and Q. H. Xu, Longitudinal polarization of hyperon and anti-hyperon in semiinclusive deep-inelastic scattering, Phys. Rev. D 79, 094018 (2009).

[47] Y. Chi, X. Du, and B.-Q. Ma, Nucleon strange $s \bar{s}$ asymmetry to the $\Lambda / \bar{\Lambda}$ fragmentation, Phys. Rev. D 90, 074003 (2014).

[48] X. Du and B.-Q. Ma, Strange quark-antiquark asymmetry of nucleon sea from $\Lambda / \bar{\Lambda}$ polarization, Phys. Rev. D 95, 014029 (2017).

[49] G. Baum et al., A New Measurement of Deep Inelastic $e-p$ Asymmetries, Phys. Rev. Lett. 51, 1135 (1983).

[50] K. Abe et al. (E154 Collaboration), Precision Determination of the Neutron Spin Structure Function $g_{1}^{n}$, Phys. Rev. Lett. 79, 26 (1997).

[51] B.-Q. Ma and A. Schafer, Parton sum rules and improved scaling variable, Phys. Lett. B 378, 307 (1996); Erratum, Phys. Lett. B380, 495(E) (1996).

[52] R. D. Field and R. P. Feynman, Quark elastic scattering as a source of high transverse momentum mesons, Phys. Rev. D 15, 2590 (1977).

[53] F. E. Close, $v W_{2}$ at small $\omega^{\prime}$ and resonance form-factors in a quark model with broken SU(6), Phys. Lett. 43B, 422 (1973).

[54] R. D. Carlitz, SU(6) symmetry breaking effects in deep inelastic scattering, Phys. Lett. 58B, 345 (1975).

[55] J. Kaur, Spin dependent distributions in the quark parton model, Nucl. Phys. B128, 219 (1977).

[56] A. Schaefer, Spin dependent structure functions for the proton and neutron, Phys. Lett. B 208, 175 (1988).

[57] B.-Q. Ma, D. Qing, and I. Schmidt, Electromagnetic form-factors of nucleons in a light cone diquark model, Phys. Rev. C 65, 035205 (2002).

[58] B.-Q. Ma, D. Qing, and I. Schmidt, Axial vector form-factor of nucleons in a light cone diquark model, Phys. Rev. C 66, 048201 (2002).

[59] T. Liu and B.-Q. Ma, Generalized form factors of the nucleon in a light-cone spectator-diquark model, Phys. Rev. C 89, 055202 (2014).

[60] J. Zhang and B.-Q. Ma, Electroweak properties of octet baryons in a light-cone quark-diquark model, Phys. Rev. C 93, 065209 (2016).

[61] Z. Lu and B.-Q. Ma, Sivers function in light-cone quark model and azimuthal spin asymmetries in pion electroproduction, Nucl. Phys. A741, 200 (2004).

[62] J. She, J. Zhu, and B.-Q. Ma, $h_{1 T}^{\perp}$ and quark orbital angular momentum, Phys. Rev. D 79, 054008 (2009).

[63] Z. Lu and B.-Q. Ma, Quark helicity distributions in transverse momentum space and transverse coordinate space, Phys. Rev. D 87, 034037 (2013).
[64] Z. Lu and I. Schmidt, T-odd quark-gluon-quark correlation function in the diquark model, Phys. Lett. B 712, 451 (2012).

[65] T. Liu and B.-Q. Ma, Quark Wigner distributions in a light-cone spectator model, Phys. Rev. D 91, 034019 (2015).

[66] S. J. Brodsky, T. Huang, and G. P. Lepage, Hadronic wave functions and high momentum transfer interactions in quantum chromodynamics, Conf. Proc. C 810816, 143 (1981).

[67] T. Huang, B.-Q. Ma, and Q. X. Shen, Analysis of the pion wave function in light cone formalism, Phys. Rev. D 49, 1490 (1994).

[68] J. Pumplin, D. R. Stump, J. Huston, H. L. Lai, P. M. Nadolsky, and W. K. Tung, New generation of parton distributions with uncertainties from global QCD analysis, J. High Energy Phys. 07 (2002) 012.

[69] F. Ringer and W. Vogelsang, Single-spin asymmetries in $W$ boson production at next-to-leading order, Phys. Rev. D 91, 094033 (2015).

[70] C. Bourrely, J. Soffer, and F. Buccella, A statistical approach for polarized parton distributions, Eur. Phys. J. C 23, 487 (2002).

[71] R. S. Bhalerao, Is the polarized anti-quark sea in the nucleon flavor symmetric?, Phys. Rev. C 63, 025208 (2001).

[72] M. Gluck and E. Reya, Phenomenology of the flavor asymmetry in the light quark sea of the nucleon, Mod. Phys. Lett. A 15, 883 (2000).

[73] D. de Florian, R. Sassot, M. Stratmann, and W. Vogelsang, Extraction of spin-dependent parton densities and their uncertainties, Phys. Rev. D 80, 034030 (2009).

[74] F. Arbabifar, A. N. Khorramian, and M. Soleymaninia, QCD analysis of polarized DIS and the SIDIS asymmetry world data and light sea-quark decomposition, Phys. Rev. D 89, 034006 (2014).

[75] D. Diakonov, V. Petrov, P. Pobylitsa, M. V. Polyakov, and C. Weiss, Nucleon parton distributions at low normalization point in the large $N_{c}$ limit, Nucl. Phys. B 480, 341 (1996).

[76] D. Diakonov, V. Petrov, P. Pobylitsa, M. V. Polyakov, and C. Weiss, Unpolarized and polarized quark distributions in the large $N_{c}$ limit, Phys. Rev. D 56, 4069 (1997).

[77] M. Wakamatsu and T. Watabe, Do we expect light flavor sea quark asymmetry also for the spin dependent distribution functions of the nucleon?, Phys. Rev. D 62, 017506 (2000).

[78] B. Dressler, K. Goeke, M. V. Polyakov, and C. Weiss, Flavor asymmetry of polarized anti-quark distributions and semiinclusive DIS, Eur. Phys. J. C 14, 147 (2000).

[79] R. J. Fries, A. Schafer, and C. Weiss, Polarized anti-quark flavor asymmetry: Pauli blocking versus the pion cloud, Eur. Phys. J. A 17, 509 (2003).

[80] E. R. Nocera, R. D. Ball, S. Forte, G. Ridolfi, and J. Rojo (NNPDF Collaboration), A first unbiased global determination of polarized PDFs and their uncertainties, Nucl. Phys. B887, 276 (2014).

[81] M. Hirai and S. Kumano (Asymmetry Analysis Collaboration), Determination of gluon polarization from deep inelastic scattering and collider data, Nucl. Phys. B813, 106 (2009). 